\title{
Sequential Combination Therapy Leading to Sustained Remission in a Patient with SAPHO Syndrome
}

\author{
C.E. Huber ${ }^{1,}$, A.G. Judex ${ }^{1, \S}$, J. Freyschmidt ${ }^{2}$, S. Feuerbach ${ }^{3}$, J. Schölmerich ${ }^{*, 1}$ and U. Müller-Ladner ${ }^{1,4}$ \\ ${ }^{I}$ Department of Internal Medicine I, University of Regensburg, Regensburg, Germany \\ ${ }^{2}$ Department of Radiology, Zentralkrankenhaus St.-Jürgen-Strasse, Bremen, Germany \\ ${ }^{3}$ Department of Radiology, University of Regensburg, Regensburg, Germany \\ ${ }^{4}$ Department of Internal Medicine and Rheumatology, Justus-Liebig University Giessen/Kerckhoff Clinic Bad \\ Nauheim, Germany
}

\begin{abstract}
The SAPHO syndrome represents a variety of clinically similar disorders with the key features of hyperostotic bone lesions in combination with chronic pustular skin disease. The respective pathophysiology of bone and joint manifestations in SAPHO syndrome is still a matter of discussion. For example it does not appear to represent reactive arthritis and HLA B27 antigen, with the latter being typically present in patients with spondyloarthopathies. Treatment of SAPHO syndrome is also not well established and consists of various antiinflammatory and antirheumatic drugs. Here, we report a female patient with active SAPHO syndrome suffering from sternal swelling of unknown origin that had been known for 10 years and a 4-year-history of severe lower back pain. Remarkable were also a typical pustulous palmar erythema associated with swelling and decreased motility of both MCP-I joints. Inflammation parameters were high with an ESR 68 $\mathrm{mm} / 1 \mathrm{st}$ hour and a CRP of $19.6 \mathrm{mg} / \mathrm{l}$. She was initially treated with rofecoxib and doxycycline, followed by sulfasalazine with only partial clinical response. Thereafter, both articular symptoms as well as cutaneous lesions responded well to a combination therapy with methotrexate and sulfasalazine. Thus, the case illustrates nicely that methotrexate in combination with another DMARD can be successfully applied to patients with long-term active SAPHO syndrome.
\end{abstract}

Keywords: Case report, combination therapy, doxycycline, methotrexate, rofecoxib, SAPHO, sulfasalazine, treatment.

\section{INTRODUCTION}

The SAPHO syndrome represents a variety of clinically similar disorders with the key features of hyperostotic bone lesions, frequently seen on the anterior chest wall, in combination with chronic pustular skin disease [1]. The acronym SAPHO (for synovitis, acne, pustulosis, hyperostosis, and osteitis) was proposed by Chamot et al. after a national survey carried out in France 1986 to evaluate the range of skin, bone and joint manifestations observed. At present, there are over 450 published cases of SAPHO syndrome.

The pathogenesis and pathophysiology of bone and joint manifestations in SAPHO syndrome remain controversial. They do not appear to represent reactive arthritis and HLA B27 antigen, which is typically present in patients with spondyloarthropathies, and is usually lacking. However, in clinical practice, SAPHO syndrome has been associated with spondyloarthropathies, especially in those cases showing sacroiliac involvement and enthesiopathies. Sonozaki et al. have proposed diagnostic criteria for the SAPHO syndrome: pustulosis palmaris et plantaris, tenderness and swelling of

*Address correspondence to this author at the Department of Internal Medicine I, University of Regensburg Medical Center, Franz-Josef-Strauss Allee 11, D-93053 Regensburg, Germany; Tel: +49 941944 7001; Fax: +49 941 944 7002; E-mail: juergen.schoelmerich@klinik.uni-r.de

${ }^{\S}$ These authors contributed equally to this paper. the sternocostoclavicular region, or in the absence of swelling, the presence of radiologic signs of hyperostosis [2]. Technetium bone scintigrams and computed tomographs are considered the most important diagnostic tests. Due to the low number of patients, treatment of SAPHO syndrome is poorly established, it consists usually of antiinflammatory agents such as NSAIDs, and corticosteroids for more severe forms [3]. Case reports have also reported the use of various DMARDs with conflicting results [4].

\section{CASE}

A 63-year-old woman was referred to our hospital for evaluation of a sternal swelling of unknown origin that had been known for 10 years. In addition, she had a 4-year-history of severe lower back pain. At time of admission, she complained about chronic pain in the lower back and in both metacarpophalangeal (MCP) I joints. She further reported episodes of loose stools and "allergies" to nonsteroidal antirheumatic drugs. Examination showed a tender swelling of her right sternoclavicular joint and a reduced motility of the complete vertebral column. A pustulous palmar erythema (Fig. 1a) associated with swelling and decreased motility of both MCPI joints was remarkable. Erythrocyte sedimentation rate was $68 \mathrm{~mm} / 1$ st hour, the $C$ reactive protein concentration was 19.6 $\mathrm{mg} / \mathrm{l}(<5)$. Immunoglobulins A and $\mathrm{E}$ were elevated, all other rheumatologic parameters including rheumatoid factor were within the normal range or negative. Conventional X-Rays and computed tomographs showed sternal hyperostotic re- 
gions with a ventrodorsal diameter of $3 \mathrm{~cm}$ and a transversal diameter of $4 \mathrm{~cm}$ (Fig. 2a). Bone scintigrams demonstrated multiple enhanced signal intensities that were consistent with pustulotic arthroosteitis (Fig. 2b). A dermatology referral confirmed that the skin eruptions on palms and hands were a manifestation of SAPHO syndrome. The clinical picture combining hyperostosis of the sternum with palmoplantar pustulosis and spondylitis fulfilled the diagnostic criteria for SAPHO syndrome as outlined above. In addition, colonoscopy revealed aphthous lesions in the ileum with histological signs of chronic inflammation.

(a)

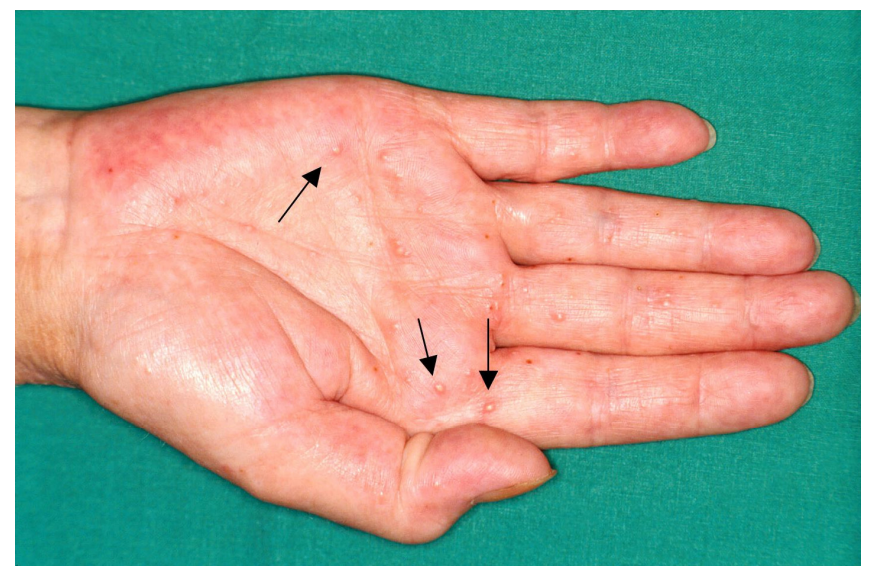

(b)

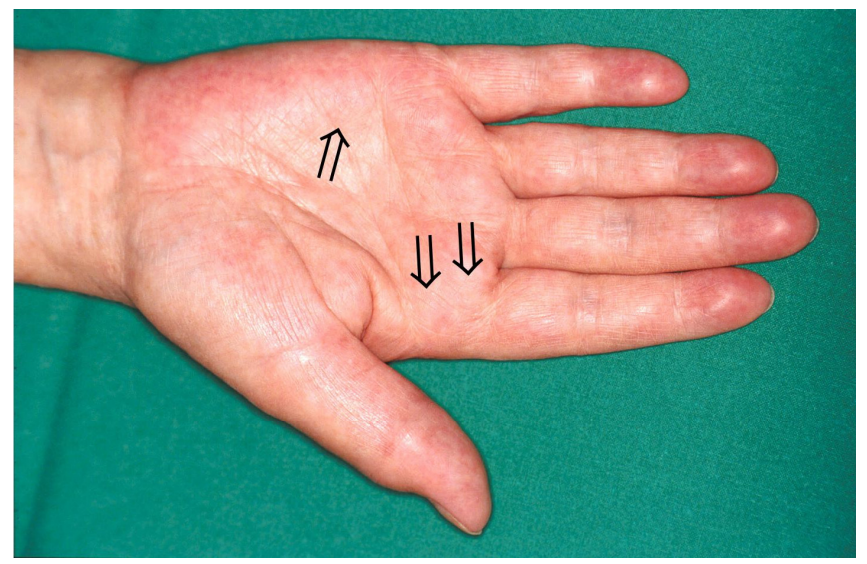

Fig. (1). (a) Typical pustulous palmar eruptions at the time of initial presentation ( $\uparrow$ ). (b) Presentation after 6 weeks doxycyline/8 weeks sulfasalazine therapy $(\Uparrow)$.

There are several reports about a possible infectious etiology of the SAPHO syndrome. Edlund and coworkers reported isolation of Propionibacterium acnes, a Grampositive rod, in a series in 7 of 15 patients from patients with SAPHO syndrome [5] and Ballare et al. have reported a sustained response to doxycycline therapy in two patients with SAPHO syndrome [6]. Therefore, we decided to start a combination therapy consisting of rofecoxib $12.5 \mathrm{mg}$ daily with doxycycline $100 \mathrm{mg}$ bid although skin cultures of the palmoplantar lesions were negative for bacteria. Sulfasalazine is recommended for the therapy of many HLA B27 antigen-negative and -positive spondylarthropathies and has shown good results for SAPHO syndrome in uncontrolled reports [7]. Although sulfasalazine as a therapeutic agent in (a)

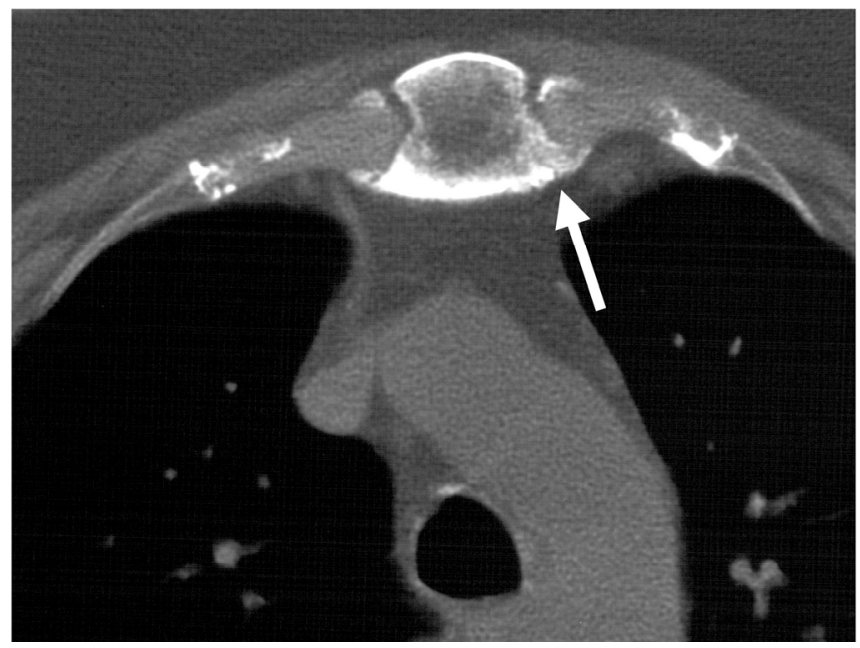

(b)

(c)

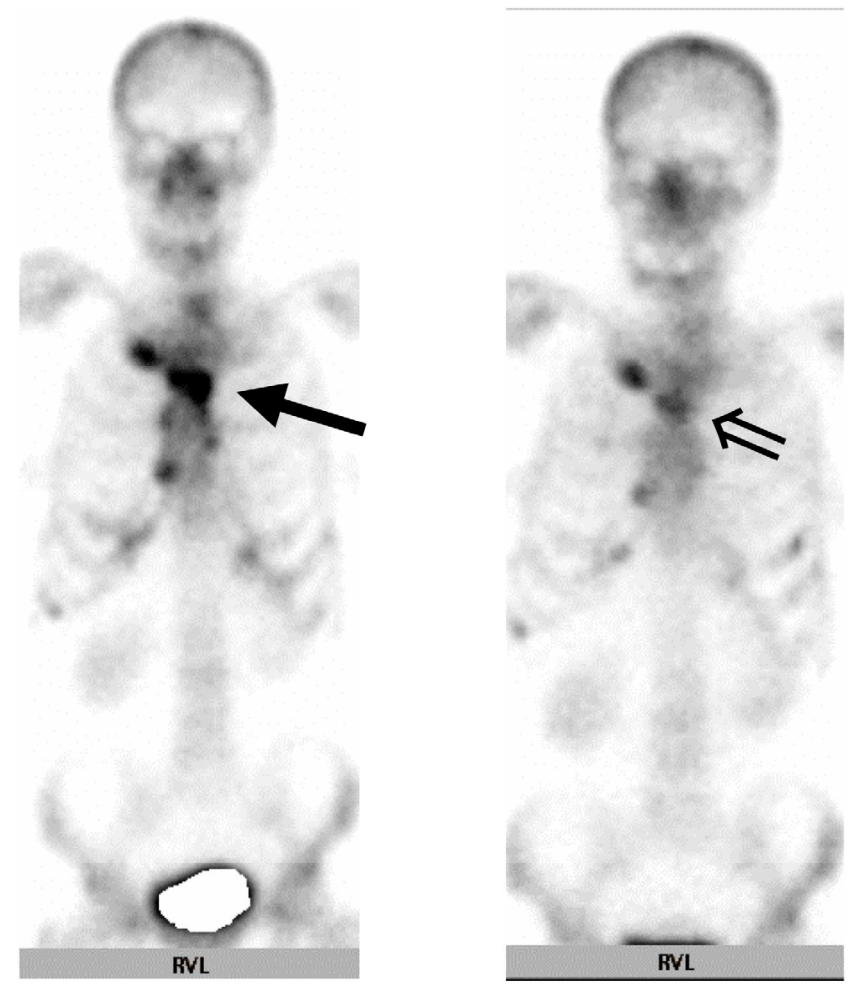

Fig. (2). (a) Axial CT scan of the sternal region reveals marked hyperostosis and osteitis of the sternum ( $\uparrow$ ). (b) Technetium-99m bone scintigram shows considerable activity in the sternoclavicular, sternocostal joints and the vertebral column ( $\uparrow$ ). (c) After 26 months of therapy, overall signal intensities have decreased substantially in the sternal region $(\Uparrow)$.

SAPHO syndrome has not been evaluated in controlled clinical trials and as other reports link SAPHO syndrome with chronic inflammatory bowel disease [8,9], we added sulfasalazine with an initial dose of $500 \mathrm{mg}$ twice a day to the patient's medication.

The patient was discharged and followed on a regular basis in our outpatient rheumatology clinic for two and a half years. The course of disease and treatment are summarized in Fig. (3). The initial treatment with doxycycline had to be 


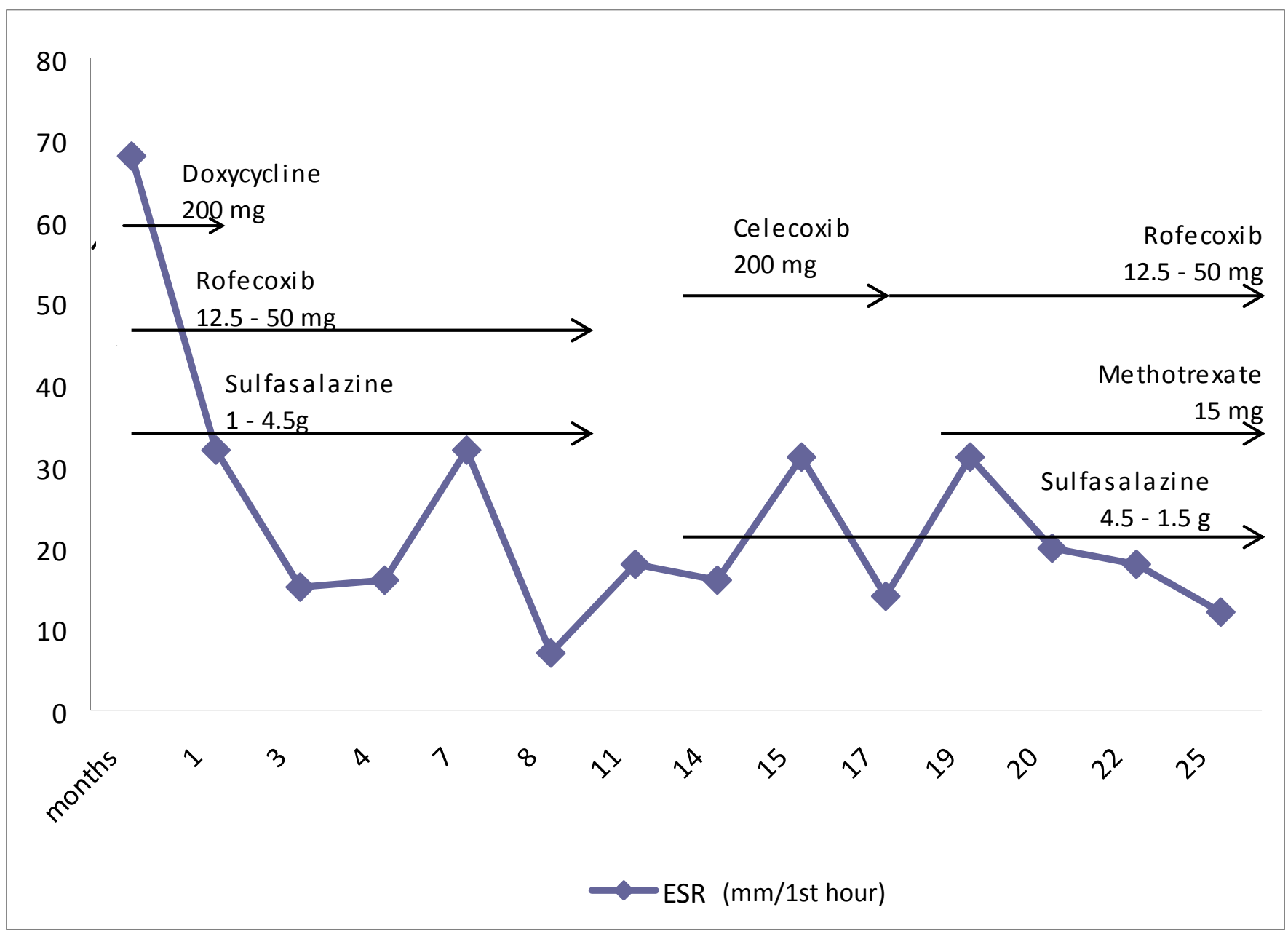

Fig. (3). Overview over treatment and disease activity. ESR values $\left(1^{\text {st }}\right.$ hour $)$ and concurrent therapy are shown for a 31 -month-period.

discontinued after 6 weeks due to oral aphthous lesions and the dose of sulfasalazine was increased to a total daily dose of $4.5 \mathrm{~g}$. Over 8 weeks, the back and joint improved markedly, skin eruptions disappeared completely (Fig. 1b) and the erythrocyte sedimentation rate (ESR) returned to $32 / 1^{\text {st }}$ hour. After 6 months under this therapy, bone scintigrams also improved markedly (Fig. 2c). A clinical and scintigraphic relapse occurred when the patient stopped taking her medication for a three month period between clinical visits. Symptoms ameliorated after starting again with sulfasalazine (Fig. 3). After 19 months, due to increased activity, methotrexate (MTX) was added to the above regimen at a dose of $15 \mathrm{mg}$ weekly (in combination with folate) and sulfasalazine was reduced to $500 \mathrm{mg}$ three times a day. For the next three years, back pain was dramatically reduced, with the exceptions of a flare-up during a urinary tract infection and a mild viral respiratory tract infection. The remaining pain in both MCP-I joints resolved after carpal tunnel surgery.

\section{DISCUSSION}

The hallmark of SAPHO syndrome is an inflammatory polytopic arthroosteits, which is characteristically sterile, although infectious agents are suspected as trigger $[5,6]$.
There are several reports about a possible infectious etiology of the SAPHO syndrome. Edlund and coworkers reported isolation of Propionibacterium acnes, a Gram-positive rod, in a series in 7 of 15 patients from patients with SAPHO syndrome [7] and Ballare et al. have reported a sustained response to doxycycline therapy in two patients with SAPHO syndrome [8]. The present case demonstrates that combination therapy with an antibiotic and a spondyloarthropathy-approved disease-modifying drug (DMARD), e.g. doxycycline and sulfasalazine, may be a successful approach for the initial therapy of this disease entity. Owing to the rare nature of SAPHO syndrome, sulfasalazine has not yet been evaluated in controlled clinical trials as a therapeutic agent. Nevertheless, Sulfasalazine is recommended for the therapy of many HLA B27 antigen-negative and -positive spondylarthropathies. Other reports link SAPHO syndrome with chronic inflammatory bowel disease [9, 10], and have shown good results for SAPHO syndrome in uncontrolled reports [11]. Consistent with promising data of Cabay and coworkers, who reported rapid improvement in a patient with pustulosis plantaris and hyperostosis of the lumbar spine after two months of therapy with MTX (7.5 mg weekly) [12], MTX facilitated in our case the reduction of the dose of sulfasalazine in a similar manner in long term therapy. 


\section{REFERENCES}

[1] Chamot AM, Benhamou CL, Kahm MF, Beraneck L, Kaplan G, Prost A. Le syndrome acne pustulose hyperostose osteite (SAPHO): resultats d'une enquete nationale. Rev Rheum 1987; 54: $187-96$.

[2 ] Sonozaki H, Azuma A, Okai K, et al. Clinical features of 22 cases with "inter-sterno-costo-clavicular ossification": a new rheumatic syndrome. Arch Orthop Trauma Surg 1979; 95: 13-22.

[3] Kahn MF. Why the "SAPHO" syndrome? J Rheumatol 1995; 22: 2017-9.

[4] Oliveri I, Padula A, Palazzi C. Pharmacological management of SAPHO syndrome. Expert Opin Investig Drugs 2006; 15: 1229-33.

[5] Edlund E, Johnsson U, Lidgren L, et al. Palmoplantar pustulosis and sternoclavicular arthro-osteitis. Ann Rheum Dis 1988; 47: 80915 .

[6] Ballara SC, Siraj QH, Maini RN, Venables PJ. Sustained response to doxycycline therapy in two patients with SAPHO syndrome. Arthritis Rheum 1999; 42: 819-21.
[7] Hayem G, Bouchaud-Chabot A, Benali K, et al. SAPHO syndrome: a long-term follow-up study of 120 cases. Semin Arthritis Rheum 1999; 29: 159-71.

[8] Kahn MF, Chamot AM. SAPHO syndrome. Rheum Dis Clin North Am 1992; 18: 225-46.

[9] Dharancy S, Talbodec N, Asselah T, et al. The synovitis-acnepustulosis-hyperostosis-osteitis (SAPHO) syndrome, a rare extradigestive manifestation of Crohn's disease: presentation of one case and review of the literature. Gastroenterol Clin Biol 1998; 22: 2403.

[10] Perez C, Hidalgo A, Olier J, Otermin I. MR imaging of multifocal spondylodiskitis as the initial manifestations of SAPHO syndrome. Am J Roentgenol 1998; 171: 1431-2.

[11] Hurtado-Nedelec M, Chollet-Martin S, Nicaise-Roland $\mathrm{P}$, et al. Characterization of the immune response in the synovitis, acne, hyperostosis, osteitis (SAPHO) syndrome. Rheumatology 2008; 47: 1160-7.

[12] Cabay JE, Marcelis S, Dondelinger RF. Inflammatory spondylodiscitis as a unique radiological manifestation of the SAPHO syndrome. J Radiol 1998; 79: 337-40.

(C) Huber et al.; Licensee Bentham Open.

This is an open access article licensed under the terms of the Creative Commons Attribution Non-Commercial License (http://creativecommons.org/licenses/by$\mathrm{nc} / 3.0 /$ ) which permits unrestricted, non-commercial use, distribution and reproduction in any medium, provided the work is properly cited. 